\title{
The rate of word integration and the overprinting paradigm
}

\author{
D. J. K. MEWHORT and A. J. CAMPBELL \\ Queen's University, Kingston, Ontario $K 7 L$ 3N6, Canada
}

\begin{abstract}
In the first experiment, the letters of words were overprinted; that is, successive letters were presented on the same location of the screen. Word identification was difficult unless the rate of presentation was slow, about 3 letters/sec. The second experiment showed that the low level of word identification did not reflect difficulty with basic letter identification. Instead, it reflected the rate at which short-term memory can accept separate items. While such results refute models of reading that stress a letter-by-letter integration, they do not test those that stress construction of higher order units. Instead, the task forces letter-byletter integration by removing spatial information, a dimension required for construction of higher order units. As a result, it does not permit integration of the correct units. The third experiment illustrated the construction and integration of supraletter units. Letter groups (either syllables or corresponding nonsyllable groups) were overprinted, a tochnique that introduces the spatial dimension. Word identification increased dramatically, and subjects were able to exploit the familiarity inherent in syllabic presentations. Thus, integration is much faster when more appropriate units can be used, and construction of the units depends on spatial information. The fourth experiment showed that the construction of supraletter units is achieved prior to short-term memory: Even though memory was able to group the material, the subjects could not use syllabic structure when syllables were isolated temporally but not spatially. The results were discussed in terms of a model describing the construction of higher order units.
\end{abstract}

In a recent paper, Mewhort and Beal (1977) described a sequence of processing stages thought to mediate word identification. Their account, like that offered by Smith and Spoehr (1974), suggests that a postidentification character buffer holds a visual-spatial representation of the character string. The buffer is thought to provide input to a scan-parse operator; the scan-parse operator, in turn, accepts the character string, derives a series of units by applying orthographic rules to the string, and passes the units to memory sequentially using a left-to-right order. Once in memory, the units are thought to serve as basic data for several processes (e.g., pronunciation, rehearsal, and lexical addressing mechanisms).

The parsing model is similar to the account offered by Smith and Spoehr (1974): Both are formalized as computer programs, both stipulate the same system architecture, and both start with the grammar described by Hansen and Rogers (1968). In contrast with the Smith and Spoehr account, we rely less heavily on pronunciation (and on translation to a phonological

This research was supported by Grant APA-318 from the National Research Council of Canada to the first author. We thank Pat Crawford and M. J. Withey for assistance in conducting experiments. We also thank G. T. Campbell and M. L. Dow for critical comments on various drafts of the work. A preliminary report of the data was presented at the meeting of the Canadian Psychological Association, Quebec, P.Q., June 1979. Requests for reprints may be addressed to either author. code) and have extended the parsing grammar by including a number of orthographic and word-level syntactic rules in the parser. Before attempting to parse, for example, our model recognized certain consonant clusters, such as "th," "ph," and "ch." Similarly, it recognized some diphthongs and includes pronunciation rules of the sort illustrated by the silent "e" in "hope." Of course, to implement the silent-e rule, one must include some decomposition rules: The latter are used to decompose a word and thereby to deal with words including a suffix, such as "love" and "lovely." Venezky's (1970) work has been particularly helpful in guiding the extensions.

We have used the extended set of rules for parsing to predict performance in free recall tachistoscopic tasks involving pseudowords of various orders of approximation to English (Mewhort \& Campbell, Note 1). Specifically, the larger the number of units produced by the parser, the lower the mean number of letters reported correctly. Predicting pseudoword performance from the parsing rules, of course, complements the parallel between word identification and pseudoword performance reported by Mewhort and Beal (1977, Experiment 1).

Models implicating derived units, called translation units by Smith and Spoehr (1974), usually stress the relation of the unit to an acoustic or phonological representation (e.g., Smith \& Spoehr, 1974, pp. 258-260). We stress, in contrast, the change from a spatial data 
representation to a temporal one. Thus, the derived units serve as data for a pronunciation mechanism; they are not, as implied by many translation-unit accounts, the product of such a mechanism. Further, because the units result from an interaction of orthographic rules with the particular character string, the nature of the units depends on the nature of the string. When applied to words, the rules yield syllables or syllable-like units. Applied to pseudowords, however, the rules produce units less like syllables. For difficult material, such as a consonant string, the rules yield single-letter units.

Our stress on data representations, both spatial and temporal, derives from earlier work on order of report in free recall (e.g., Hearty \& Mewhort, 1975; Mewhort, 1974; Mewhort \& Beal, 1977, Experiment 1). Similarly, we emphasize rules for constructing units rather than the units themselves and acknowledge that several types of unit are used. In addition to escaping endless debate about the unit, a concept more appropriate to a passive filter account, the model avoids a difficulty often associated with translation-unit accounts: By linking the unit too closely to a phonological representation, most translation-unit accounts have difficulty simulating performance on hard-to-pronounce material. Like other derived-unit models, however, our account stipulates that the internal representation of a word, the representation used for semantic operations, involves the derived units.

Kolers (1972) has suggested, in contrast, a more direct route to meaning. His account was motivated, in part, by early studies discrediting a letter-by-letter integration process (e.g., Huey, 1908/1968; Kolers \& Katzman, 1966). In the Kolers and Katzman (1966) experiments, for example, successive letters of a word were printed on the same spot on a screen, and the exposure time of the letters was varied over a wide range. Word identification was remarkably difficult unless the rate was slow (i.e., approximately $3-4$ letters/ sec). Consequently, Kolers argued that integration does not offer a viable account of reading; it is too slow to support reading faster than 35 words/min (e.g., Kolers, 1972).

Although the scan-parse model suggests that word identification is mediated by an integrative mechanism, the letter overprinting experiments do not provide a reasonable test of that aspect of the account. Letter overprinting forces the subjects to assemble separate letters into a word. While the scan-parse account requires an initial representation of the letters, the units assembled into a word are products of the scan-parse operator. Thus, letter overprinting examines integration of the wrong units. Moreover, the paradigm prohibits construction of the appropriate units: The scan-parse mechanism requires horizontal spatial information (e.g., Hearty \& Mewhort, 1975; Mewhort \& Beal, 1977, Experiment 3); letter overprinting deprives it of a necessary spatial context. Thus, the technique not only examines the wrong units, it also preciudes examination of appropriate ones.

If the operator cannot function, the material passed to memory does not benefit from the data transformation ordinarily accomplished by the parse mechanism. As a result, it should be passed in a form less suitable to subsequent memory operations (see Mewhort, 1974, for an example). Thus, because the model predicts a slow rate of integration in their paradigm, the Kolers and Katzman (1966) estimate for the speed of integration with letter overprinting is consistent with the scanparse account.

While letter overprinting does not provide a fair test of the integration aspect of the scan-parse model, a simple extension of the technique would provide a means to examine that process. Suppose that letter groups, oriented horizontally, were presented succes. sively on the same spot. Because appropriate spatial information would be provided, the scan-parse operator should be invoked, and the rate of input to memory should increase. The extent of the increase should depend on the nature of the letter groups. Because the operator applies orthographic knowledge to derive units, performance should increase more when the structure of the groups is consistent with the knowledge than when it is not.

\section{EXPERIMENT 1}

The experiments presented here explore predictions for the overprinting paradigm derived from the scanparse model. The first experiment, a simple letter overprinting task, was designed to replicate aspects of the Kolers and Katzman (1966) study. A replication is necessary because our display procedure involves some potentially serious technical differences. Kolers and Katzman used a motion-picture technique to present black letters on a light background. The rate of presentation was varied by changing the exposure time of successive displays. Our technique involves computer-generated characters presented as a bright display on a dark cathode-ray tube, and the rate of presentation was manipulated for fixed-duration displays by varying the interletter interval.

\section{Method}

Subjects. The subjects were eight undergraduates enrolled in introductory psychology courses at Queen's University. Participation in the experiment fulfilled part of the course require ments. All subjects reported normal or corrected-to-normal vision.

Materials and Apparatus. The stimuli were the 80 most frequent eight-letter words listed by KuCera and Francis (1967) that do not include repeated letters. The materials were displayed on a Tektronix point-plot display monitor (Model 604, supplied with P4 phosphor) controlled by a minicomputer (PDP-8/e). The monitor was located in a partially darkened room adjacent to the room housing the computer.

Each letter, shown in uppercase, was defined in a 5 by 7 matrix and subtended a visual angle of about $15 \mathrm{~min}$ by 
$21 \mathrm{~min}$. See Mewhort (1978) for details of the display algorithm.

Procedure. For each trial, a fixation dot was presented at the center of the monitor. Following a verbal "ready" signal, the word was presented one letter at a time. The letters of the word were presented in spelling order; that is, the first letter shown was that normally printed at the left, the second was that normally printed second from the left, and so forth. Each letter was centered at a point about $40 \mathrm{~min}$ diagonally above and to the right of the fixation point. All were presented for $5 \mathrm{msec}$, and on each trial the interletter interval was one of 50,150 , 250 , or 300 msec.

The subjects were informed that all stimuli were words, and they were asked to identify the words if possible. If a word report was not possible, however, the subjects were requested to report as many letters as possible.

Design. Each subject received 80 trials, 20 replicates at each of the four interletter intervals. Across subjects, each word was used equally often at each of the interletter intervals, and the order in which the trials were administered was randomized independently for each subject.

\section{Results and Discussion}

The responses were scored in three ways. First, we tallied the number of words identified correctly. Second, for cases involving a failure to identify the word, we tallied the number of letters reported correctly. For the latter cases, a measure of order of report was calculated by computing the rank correlation (tau) of position in the report with time of arrival on the display. Thus, a tau of +1.0 indicates a report in the order of arrival on the display; a -1.0 indicates a report in the opposite order. The score is not defined unless at least two items have been reported correctly. The expected value of the measure is zero, and the score is independent of the accuracy of report.

Figure 1 summarizes the data. The top panel shows both the proportion of words identified correctly and the proportion of letters reported correctly, given a nonword report. Both scores are expressed as a function of interletter interval. The middle panel shows the mean order-of-report score (tau) as a function of interletter interval; the bottom panel shows letter performance as a function of both interletter interval and temporal position.

Word identification increased in a linear fashion across the 50- to $250-\mathrm{msec}$ range of interletter intervals, but there was little increase from 250 to $300 \mathrm{msec}$ $[F(3,21)=52.30, p<.001]$. Similarly, letter performance given a failure to identify the word increased linearly across the range of interletter intervals $[F(3,21)=57.04, p<.001]$.

In general, the subjects tended to report in the order of presentation at all interletter intervals. Nevertheless, reducing the presentation rate increased the trend $[F(3,21)=21.56, p<.001]$.

As is shown in the bottom of Figure 1, at the $50-\mathrm{msec}$ interletter interval, report showed no primacy effect but a marked recency effect. At all of the slower rates, however, performance showed both a primacy and a recency effect; that is, it took the asymmetric U-shape associated with serial recall phenomena.
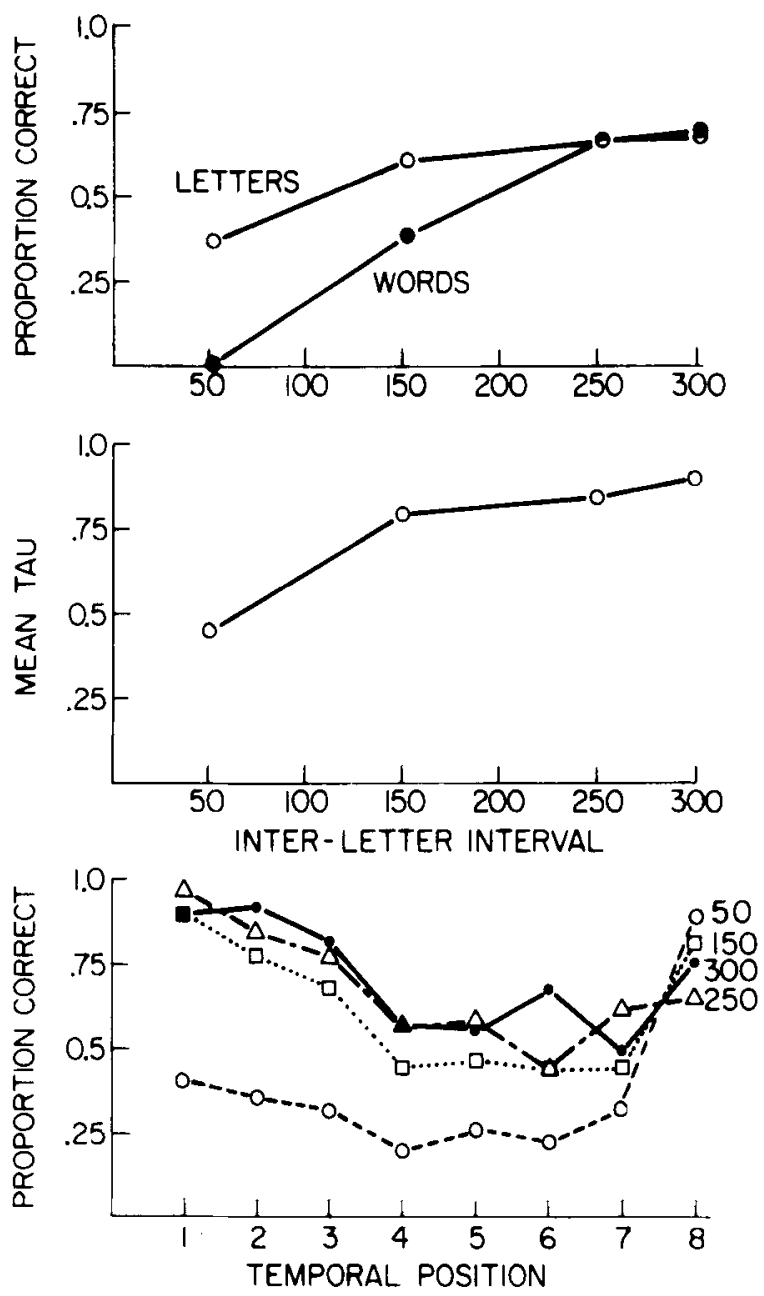

Figure 1. Overprinting: The top panel shows both the proportion of words reported correctly and the proportion of letters reported correctly given a nonword report. Both are expressed as a function of interletter interval. The middle panel shows the mean tau (order-of-report score) for letter reports as a function of interletter interval. The bottom panel shows the proportion of letters reported corrctly as a function of the interletter interval and the temporal position in the input stream.

Letter overprinting includes two straightforward limits to performance. Masking is inherent in the paradigm and may limit basic character identification. Given adequate character identification, however, the technique requires memory to accept letters at a fast rate and, if they are to be assembled into a word, to keep track of their order of entry.

At an interletter interval of $150 \mathrm{msec}$ or more, character identification is not the major problem: Identification of the first character, the one subjected to the greatest amount of backward masking, was quite good. Thus, the technique seems to stress short-term memory by requiring an overly fast input speed and/or by overloading the ordering capability. In this connection, the order-of-report data are relevant. Slowing the rate of presentation increased the trend to report in a consistent order. Presumably, that increase reflected an 
increase in the subjects' ability to keep track of the order of entry to memory as the rate of presentation was slowed. Finally, the subjects' description of the task deserves comment: The subjects reported that, regardless of the rate of presentation, they could see all of the letters but were unable to remember them. Their description is reminiscent of Sperling's (1960) demonstration: We can see more than we can recall.

In summary, the results are remarkably similar to those reported by Kolers and Katzman (1966): Word identification is greatly impaired unless the rate of presentation is slowed to about 4 letters/sec. Thus, the results confirm those reported by Kolers and Katzman and indicate that our changes in experimental technique do not introduce important differences.

\section{EXPERIMENT 2}

As noted, we think that the low performance at fast rates of presentation reflects a limit to the rate at which we can integrate successive letters. In other words, the visual system can resolve the letters, but short-term memory cannot accept separate items passed at high speed. On the other hand, the paradigm includes ample opportunity for both forward and backward masking. If fast rates of presentation exceed the temporal resolution of the visual system, the low performance associated with such rates could reflect loss of basic character information rather than an inability to integrate the characters.

Several aspects of the first experiment are easier to explain with the integration hypothesis. The masking argument, for example, has trouble explaining why a primacy effect occurred at all but the fastest rate: If limitations to character information reduce word identification at intermediate speeds, why did the letter scores show such a strong primacy effect? Similarly, the integration view suggests that slower speeds increase memory's ability to keep track of the order in which the items have been accepted (the time of arrival). The order-of-report data seem to reflect such an ability. The masking hypothesis, in contrast, suggests that more letters would be available, but it is mute about how they are organized.

Nevertheless, the two hypotheses are not mutually exclusive. Accordingly, the second experiment was designed to assess the masking hypothesis independently. Subjects were shown materials in the same way as before, but the task was changed. Instead of a word identification/free recall procedure, the subjects were required to indicate whether or not a designated letter had been presented. To the extent that performance in the first experiment reflects a limitation to basic character information, performance in the second should also reflect that difficulty; that is, it should parallel performance in the first. If performance in the first experiment reflects the rate at which memory can accept separate items, however, the parallel should not appear: Because the second experiment does not require memory to accept all of the material, it should reflect character identification processes unfettered by memory limitations. Thus, if performance in the first task reflects the rate at which memory can accept separate items, performance in the second should yield high levels of performance at the same rates of presentation that showed poor performance in the first.

\section{Method}

Subjects. The subjects were 30 undergraduates enrolled in introductory psychology courses at Queen's University. Participation in the experiment fulfilled part of the course requirements. All subjects reported normal or corrected-to-normal vision, and none had participated in the earlier study.

Materials and Apparatus. The stimuli and apparatus were the same as in Experiment 1.

Procedure. On each trial, a fixation dot appeared at the center of the screen. When ready, the subject pushed a button to initiate the trial. To start the trial, a single target letter, centered on the screen, was presented for $2 \mathrm{sec}$. After a $300-\mathrm{msec}$ pause, a word was presented one letter at a time, as in Experiment 1.

For half the trials, the designated letter was not in the word. A target letter was obtained in such cases by searching a list of second-order pseudowords for a letter not present in the word. Because such pseudowords represent each letter with its correct frequency in English, the negative targets could not be distinguished on the basis of letter frequency.

The subjects were required to indicate whether or not the designated letter had been presented. They indicated their choices by pushing one of two buttons. As in the preceding study, the subjects knew that each trial involved a word. Finally, the subjects were told that they could not predict the correct response from the preceding trial(s).

Design. Each subject received 160 trials, 80 positive trials and 80 negative trials. Each word was used on both a positive trial and a negative one. For the positive trials, there were 10 replicates for each temporal input position.

Three groups of subjects were used. The groups differed in the interletter interval between successive letters; the values for the groups were 50,125 , and $200 \mathrm{msec}$. Across subjects within a group, each word was probed equally often at each temporal input position. For each subject, the order of the trials was determined randomly.

Before starting the experiment, each subject was given 20 practice trials using different material. The practice was administered at the same rate as the trials to follow.

\section{Results}

The responses were scored by tallying the number of correct responses for each subject. ${ }^{1}$ At the 50 -msec interletter interval, performance was moderately good; the proportion of responses correct was .752 . (The hit and false alarm proportions were .734 and .228 , respectively.) With an increase in interletter interval, performance improved dramatically, but there was little difference between performance at 125 and at $200 \mathrm{msec}$; the proportions correct were .932 and .969 , respectively. (The hit and false alarm proportions were .931 and .066 , and .980 and .041 , respectively.) Analysis of variance on the number of correct responses showed a significant main effect of interletter interval $[F(2,27)=$ 
$80.58, p<.001]$. A comparison of performance at $50 \mathrm{msec}$ against that for the other two was highly significant $[F(1,27)=156.92, p<.001]$, but the contrast comparing performance at the 125- and 200-msec interletter intervals was not significant.

In addition to the main analysis, the positive trials permitted an analysis in terms of temporal position. Performance at the 50-msec rate showed both a primacy and a recency effect, but performance at the slower rates was both flat across temporal positions and at ceiling.

\section{Discussion}

The results of the second experiment contrast sharply with those of the first. In the first experiment, both word identification and letter scores increased linearly with increases in interletter interval to an asymptote at an interletter interval between 150 and $250 \mathrm{msec}$. The second experiment showed, however, that letter recognition reaches ceiling with an interletter interval of only $125 \mathrm{msec}$. Clearly, subjects can identify letters at rates that do not permit good retention.

Given the high level of letter identification at the 125 -msec interletter interval, it does not appear that masking was the major factor limiting performance in the first experiment. Instead, the low performance in that experiment must have reflected memory limitations rather than a loss of basic character information. While the contrast between the two experiments points to a memory limitation, the limitation is not a simple memory span (limited-capacity) problem: The word identification performance doubled over the 125- to 300-msec range of interletter intervals. Thus, the limitation is on the rate of encoding into memory; faster rates of presentation prohibit entry to memory, even when character identification is possible.

Work reported by Travers (1973) reinforces both the results described for Experiment 1 and the interpretation favoring the integration over the masking hypothesis: Travers used a single-letter overprinting task along with two related conditions. In the latter cases, successive letters were spread across a horizontal row, and in one of the cases, the letters were followed by a mask. ${ }^{2}$

First, consider the empirical support: Travers (1973) used a computer-controlled cathode-ray tube display. His single-letter overprinting condition yielded word recognition scores very similar to those shown in Figure 1 (top panel). Thus, both his experiment and the present results agree with those obtained by Kolers and Katzman (1966) using more conventional displays.

Second, consider the theoretical point. The spread cases offer converging evidence by illustrating the effect of masking on word integration. Unfortunately, Travers' (1973) mask manipulation was somewhat complicated: He delayed the mask by either one or two character times and thereby changed both the stimulusonset asynchrony (SOA) of overlapping characters and the metacontrast relation of the mask character to its adjacent letters. Nevertheless, when subjects were shown words and asked to identify them, introducing the mask reduced performance, and delaying the mask reduced the impact of the mask. When subjects were shown random letters (and asked to report as many as possible), however, the masking manipulation had little, if any, effect: About $65 \%$ of the letters were reported correctly regardless of the mask.

In terms of the present argument, Travers' (1973) random-letter task shows that a mask has essentially no effect on basic character identification. Under the same basic visual conditions, however, his word experiment showed a major effect of the mask. Given the random-letter results, the effect of the mask could not reflect basic visual processing. Instead, we suggest it reflects the effect of masking on word integration. Thus, the contrast between the two tasks indicates that with an SOA of $50 \mathrm{msec}$ or longer, masking has a relatively greater effect on integration processes than on basic character identification.

\section{EXPERIMENT 3}

The next experiment introduced a spatial dimension into the overprinting paradigm. Words were segmented into letter groups; each group was either a syllable or a nonsyllable. The letters of each group were presented simultaneously, but the groups were overprinted. Because the display contains the horizontal dimension, the scan-parse mechanism should parse each group and pass the resulting units to memory.

Presentation of syllables preserves several characteristics of the visual data normally available under whole-word conditions. Syllabic presentations should not separate, for example, either consonant clusters or diphthongs. As a result, syllabic presentation should permit the parse mechanism to operate in a more normal fashion than would be the case for nonsyllabic presentations, particularly nonsyllabic groups that break letter clusters. Normal parsing, in turn, should yield both fewer and more familiar units than the corresponding nonsyllable case.

The point is not that syllables will be passed to memory directly. A whole-word presentation includes information about the position of both letter clusters and vowels within the word, and position information is used by the parser. Accordingly, even syllabic presentation should yield a parsing unlike that of a corresponding whole-word presentation. Nevertheless, because the spatial information has been reinstated, parsing should occur. As a result, the material should be converted to a form suitable for short-term memory operations. Presumably, conversion to an appropriate form helps to overcome the input rate and ordering limits illustrated by the first experiment. Thus, the syllable condition should yield a higher level of word identification than 
the nonsyllable one. In addition, because the scanparse mechanism packages the material, performance should be better for either condition than in the first experiment.

\section{Method}

Subjects. The subjects were 12 undergraduates at Queen's University. Participation fulfilled part of a course requirement. All subjects reported normal or corrected-to-normal vision, and none had participated in the earlier experiments.

Apparatus and Materials. The equipment was the same as that used in the previous experiment. The materials, the same words used by Mewhort and Beal (1977, Experiment 2), were 144 eight-letter three-syllable words. The syllable boundaries were taken from a standard dictionary (Avis, 1973).

The words were divided into four boundary sets defined by the two positions marking their syllable boundaries: The boundaries followed Letters 2 and 4, 3 and 5, or 3 and 6 .

Procedure. On each trial, a central fixation point was presented, and when ready, the subject pushed a button to start the trial. A word was presented in three successive and overprinted letter groups. Each group was displayed for $5 \mathrm{msec}$, and the intergroup interval was 50,125, or $200 \mathrm{msec}$. Each group was centered about the fixation point. The visual angle of the letter group depended on the number of letters in it, with the maximum angle reaching $1 \mathrm{deg} 8 \mathrm{~min}$.

Each subject received one of two conditions. In one, the letter groups were syllables; in the other, nonsyllabic groups were used. Six subjects received each condition; the subjects were assigned randomly to the conditions.

When nonsyllabic groups were presented, the group boundaries were determined by switching to syllable boundaries of the words in another boundary set. The various boundary sets were used equally often both within and across the two groups of subjects.

Each subject received 144 trials, 48 replicates at each intergroup interval. Across subjects, each word was used equally often at each intergroup interval. The order in which the trials were administered was randomized independently for each subject.

The instructions were the same as in the previous experiment. As before, each subject received 20 practice trials with different materials.

\section{Results}

Figure 2 shows the number of words reported correctly as a function of the intergroup interval and the syllable-nonsyllable conditions. As is clear in the figure, increasing the intergroup interval increased word identification for both conditions. For the syllabic case, both the linear and the quadratic components of the trend were significant $[F(1,5)=101.16, p<.001$, and $F(1,5)=44.66, p<.001$, respectively]. For the nonsyllabic group, only the linear component was significant $[F(1,5)=92.74$ (linear), $p<.001$, and $F(1,5)=1.17$ (quadratic), $p=.33$ ]. Finally, performance in the syllabic case was better than that in the nonsyllabic case $[\mathrm{F}(1,10)=17.13, \mathrm{p}<.001]$.

Although the syllabic case yielded better word identification than the corresponding nonsyllabic group, the pattern of errors across the two groups was similar. The proportion of the errors due to false alams (i.e., incorrect word reports), for example, was .34 and .32 for the syllabic and nonsyllabic groups, respectively.

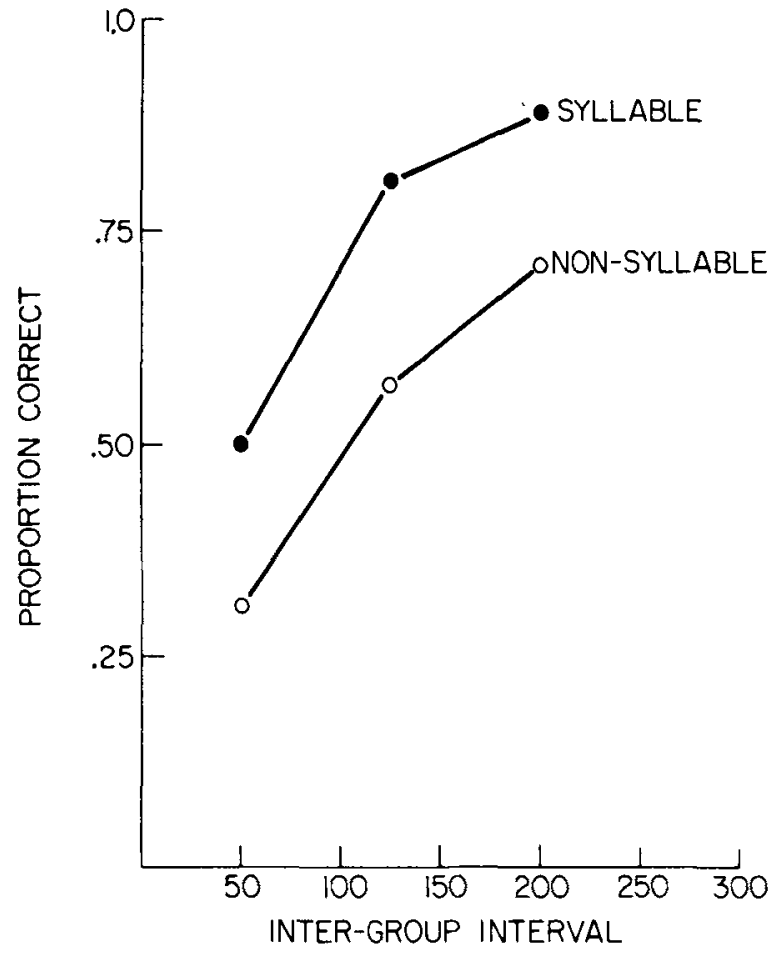

Figure 2. Group overprinting: Proportion of words identified correctly as a function of intergroup interval and syllablenonsyllable letter grouping.

For both groups, the bulk of the incorrect word reports were of about the correct length (i.e., seven to nine letters). Also, the proportion of letters correct for nonword reports was similar for the two groups. For the syllabic group, the proportions were $.57, .69$, and .75 for the 50-, 125-, and 200-msec intergroup intervals, respectively. The corresponding values for the nonsyllabic group were $.57, .66$, and .70 . Unfortunately, because of the small and unequal number of trials contributing to the proportions, a formal statistical analysis would not be meaningful.

\section{Discussion}

Consistent with the scan-parse account, introducing the horizontal dimension altered performance dramatically. First, introducing the dimension permitted subjects to exploit syllabic presentation. Second, regardless of the structural grouping, the level of performance was considerably better than the best level found with single-letter overprinting. With a $200-\mathrm{msec}$ intergroup interval, for example, syllabic overprinting yielded approximately $90 \%$ accuracy, but with a $300-\mathrm{msec}$ interletter interval, single-letter overprinting yielded only $70 \%$ accuracy, the same value obtained with nonsyllabic group overprinting at $200 \mathrm{msec}$.

The extent of the benefit of the horizontal dimension deserves emphasis. In the first experiment, low levels of word identification were associated with fast rates of presentation, that is, with rates offering minimal time 
per letter. The time per letter in the third experiment, even though it yielded better word identification, was considerably shorter than that in Experiment 1. For the slowest letter overprinting case, the duration of the whole display was $2.140 \mathrm{sec}$; for the slowest group overprinting case, the corresponding time was $.415 \mathrm{sec}$, a ratio in excess of $5: 1$. Indeed, the total presentation time for the slowest group case was about the same as that for the fastest letter case $(.415$ and $.390 \mathrm{sec}$, respectively).

Further, even though it yielded a relatively good level of word identification, at the fastest speed, group overprinting may well have involved some masking effects. Certainly, it was within the temporal range that showed such effects in both the first and the second experiments. Even with such difficulties, however, performance with syllabic presentation at the fastest rate rivaled the best achieved under letter overprinting conditions at any speed.

The high level of performance with group overprinting calls into question Kolers' (1972) estimate of the rate of serial integration. He argued that serial integration is too slow to support reading faster than about 35 words/min. We suggest, however, that his estimate is based on an inappropriate paradigm: Letter overprinting does not permit subjects to derive units and thus forces use of a slow route to memory. By introducing appropriate spatial information, the third experiment enabled subjects to derive units, a point evident from the advantage of syllabic groups. The units, in turn, provided a faster input to memory. Thus, the present data suggest an estimate for the rate of serial integration much higher than that indicated by Kolers.

\section{EXPERIMENT 4}

Our interpretation of the third experiment focuses on the scan-parse mechanism. Briefly, we suggest that it accepts the parallel letter array, constructs a verbaltemporal unit(s), and passes the unit(s) to memory. In terms of the architecture of the system, our account places the unit-construction mechanism after letter recognition but before entry to short-term memory.

The third experiment illustrated the use of supraletter units. In contrast with the present interpretation, however, one might argue that the units were constructed by short-term memory rather than by a device before it. The final experiment was designed to address that objection.

The experiment was a letter overprinting task similar to the first experiment, but it included a pause in the input stream. Like the manipulation in the third experiment, the pause was placed either between syllables or within a syllable.

One can develop two sets of predictions for the experiment. First, suppose that short-term memory is responsible for exploiting syllabic structure: If the pause is an effective manipulation, that is, if it is large enough to force an appropriate grouping, short-term memory should be able to take advantage of syllabic structure. Thus, if short-term memory is responsible for exploiting syllabic structure, a pause after a syllable should yield a clear advantage over one within a syllable.

Second, consider predictions derived from the scanparse account: The task isolates syllables temporally but does not include the spatial information thought to permit the scan-parse mechanism to operate. Accordingly, performance should be limited, as in the first experiment, by the slow input to memory. Introducing a pause early in the input stream might permit memory to "catch up" to the rapid input, but because the unit-construction mechanism is not involved, it should not be possible to exploit the syllabic structure. Thus, a pause after a syllable should be equivalent to one within a syllable.

\section{Method}

Subjects. The subjects were 16 undergraduates enrolled in introductory psychology courses at Queen's University. Participation fulfilled part of a course requirement. All subjects reported normal or corrected-to-normal vision, and none had participated in the previous studies.

Materials and Apparatus. The apparatus and the visual details were the same as those in Experiment 1. The stimuli were 48 two-syllable eight-letter words. The words were divided into three sets on the basis of their syllable boundaries. The boundaries followed Letter 3, 4, or 5. As before, Avis (1973) was consulted to determine the syllable bounds.

Procedure. On each trial, a central fixation dot appeared. When ready, the subject pushed a button to initiate the trial. Each letter was presented for $5 \mathrm{msec}$, and the interletter interval was $50,130,210$, or $290 \mathrm{msec}$. The interletter interval following Letter 3, 4, or 5, however, was replaced by a 500-msec pause. Each pause position was used equally often. On half of the trials, the pause split the word between syllables, and on the remaining trials, the pause occurred within a syllable. The subjects received 20 practice trials involving different materials; the instructions were identical to those in Experiment 1.

Design. Each subject received 96 trials, four replicates for the conditions defined by the factorial combination of three variables, interletter interval $(50,130,210$, and $290 \mathrm{msec})$, pause position (after Letter 3,4 , or 5), and syllable condition (pause between or within syllables). The 96 trials were split into two blocks of 48 , and each of the 48 words was used once within a block. Across subjects, each word was used equally of ten in both syllable conditions and in all four interletter intervals. The order in which the words were administered was determined randomly within each block by subject combination.

\section{Results and Discussion}

The responses were scored in two ways. First, we tallied the number of words identified correctly. Second, for the cases involving incorrect or nonword reports, we tallied the number of letters identified correctly. Figure 3 shows the proportion of words identified correctly as a function of pause location and interletter interval.

As is shown in Figure 3, increasing the interletter interval increased word identification $[\mathrm{F}(3,45)=48.65$, $\mathrm{p}<.001]$. In addition, word identification was better 


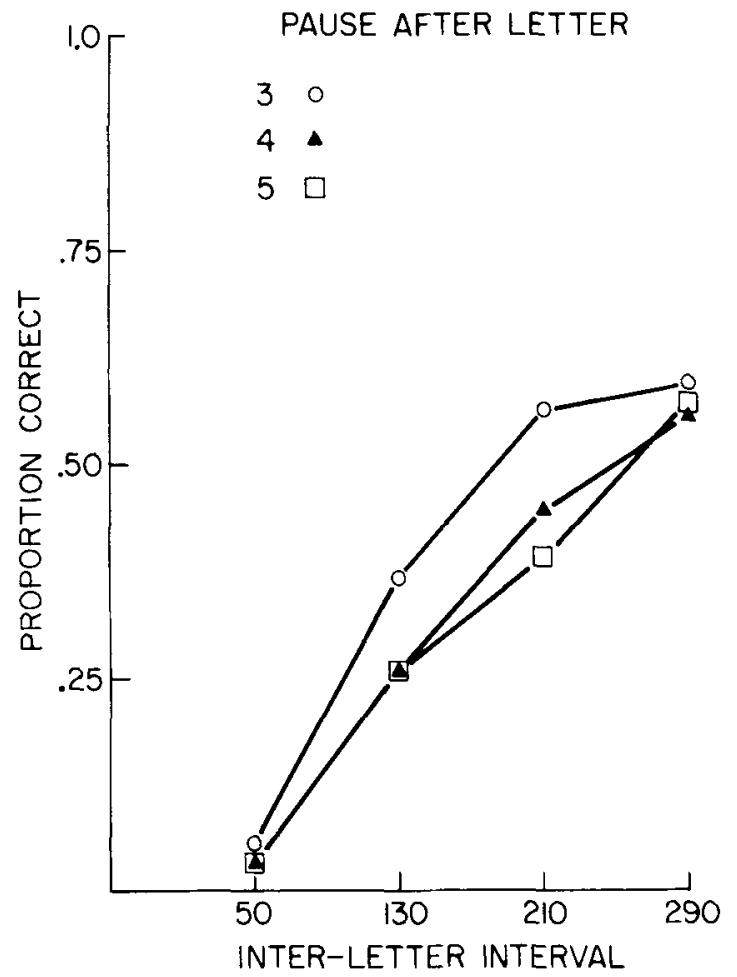

Figure 3. Letter overprinting with a pause: Proportion of words reported correctly as a function of pause location and interletter interval. with a pause after Letter 3 than with one after the other positions, but there was little difference between a pause after Letter 4 and a pause after Letter 5 $[F(2,30)=7.21, p<.01]$. Thus, a pause early in the input appears to permit memory to catch up.

The proportion of words reported correctly was .34 with a pause between syllables and .35 with the pause within a syllable $[F(1,15)=.11, p=.75]$. The difference was not significant; further, none of the interactions involving the syllable condition approached significance. The latter point is particularly interesting in light of the small benefit of a pause after position three: The failure of the interaction of pause position with the syllable. nonsyllable manipulation indicates that the benefit of an early pause extended to both kinds of presentation.

Finally, word identification was better on the second block of 48 trials; the proportion of words identified correctly was .27 and .41 , respectively $[F(1,15)=27.56$, $\mathrm{p}<.001]$. None of the interactions involving the block factor was significant.

As in the first experiment, when subjects could not report a word, they were asked to report as many letters as possible. Figure 4 shows the proportion of letters reported correctly as a function of interletter interval, pause location, and temporal position. Because word identification was not affected by the syllable-nonsyllable manipulation, the letter-report data were collapsed

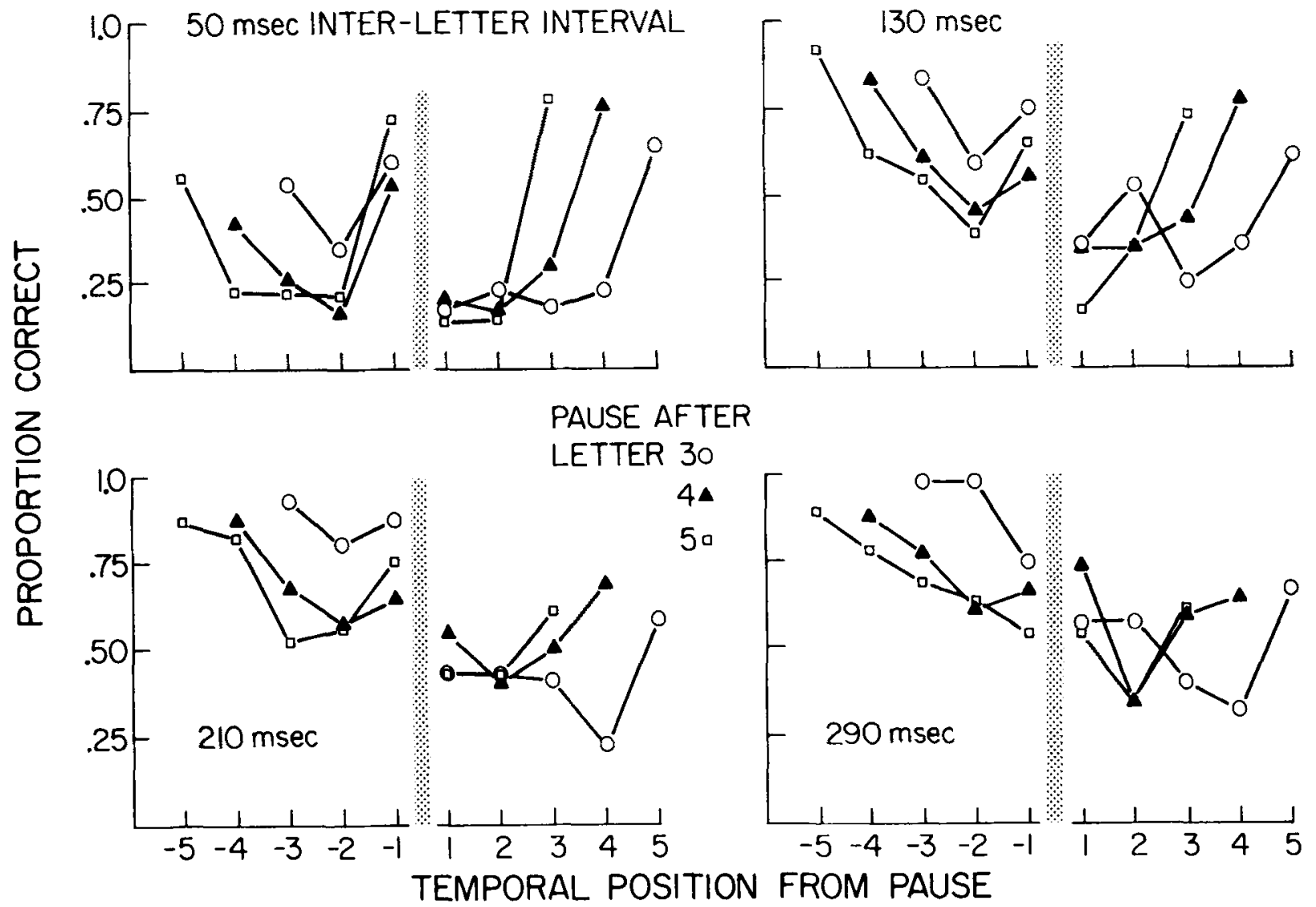

Figure 4. Letter overprinting with a pause: Proportion of letters reported correctly given a failure of word identification as a function of pause location, interletter interval, and temporal position in the input stream. 
across that factor. To clarify the figure, the data are presented as a function of temporal position relative to the pause: The location of the pause is indicated in the figure by the vertical bar.

Although introducing a pause in the input stream did not permit subjects to exploit syllabic structure, it had a major impact on letter report, given a failure to identify the word. As is shown in Figure 4, the pause split the report into two parts: In general, report before the pause showed both a primacy and a recency effect; report after the pause tended to show only a recency effect. The latter trends were absent at the slowest rate of presentation. At the slowest rate, however, because word identification was relatively high, the number of trials in the analysis was relatively small. Also, the pause represented a proportionally smaller deviation from the interletter interval. Nevertheless, taking the corresponding data from the first experiment as a reference (Figgure 1 , bottom panel), it is clear that the pause permitted the subjects to group the material.

In association with the third experiment, the pattern of results makes it clear that supraletter units are achieved prior to short-term memory. To illustrate the point, suppose instead that short-term memory were responsible for constructing supraletter units. If shortterm memory were responsible for constructing derived units, why did the subjects fail to exploit syllabic structure in Experiment 4 when they did so in Experiment 3? One cannot argue that they failed to group the material and, therefore, did not use the syllabic structure; the evidence is clear that subjects did group the material (Figure 4). Indeed, the evidence for grouping in short-term memory underscores the failure of a syllable effect: Although short-term memory can group material, it did not exploit syllable structure. Presumably, syllabic structure is used prior to shortterm memory. In particular, we suggest that supraletter units are produced by the scan-parse mechanism.

The suggestion that a mechanism prior to shortterm memory derives units may seem to conflict with the organizational capabilities associated with shortterm memory. Short-term memory is thought, for example, to permit information-compression operations, called "chunking" by Miller (1956). Presumably, chunking is a relatively slow operation, one that has general applicability. The scan-parse operator, in contrast, is a high-speed device, which, because it uses specialized orthographic knowledge, has a limited applicability; it is a reading-specific routine. Thus, instead of a conflict between the two concepts, we see a complementary relation. Assuming units are constructed prior to shortterm memory, the major advantage of such units lies in the flexible processing they permit once passed to shortterm memory. Well constructed units should enjoy an advantage by virtue of simple pronounceability. Nevertheless, the point remains: Construction of the unit precedes its use.

\section{CONCLUSIONS}

Experiment 1 replicated the Kolers and Katzman (1966) result: With letter overprinting, word identification was difficult unless the rate of presentation was very slow. Experiment 2 showed that the low performance in the first experiment did not reflect loss of basic character information. Instead, it reflected a limitation in the rate short-term memory can accept separate items; letter overprinting forces letter-by-letter integration by removing the spatial information required for construction of derived units.

Experiment 3 illustrated construction of units by introducing a horizontal spatial dimension. Word identification increased dramatically, and the subjects were able to exploit the orthographic regularity of syllabic presentations. Thus, integration is much faster when appropriate units can be used, and the construction of such units depends on the availability of suitable spatial information.

Experiment 4 showed that the units are constructed prior to short-term memory. When a pause was introduced into the letter overprinting paradigm, letter report showed strong evidence of grouping in short-term memory. Because the paradigm prohibits construction of units prior to short-term memory, however, word identification was difficult at fast rates of presentation, and the subjects were unable to exploit the orthographic regularity of syllabic structure.

Experiments 3 and 4 are similar to studies reported by Mewhort and Beal (1977, Experiments 2 and 3). In their experiments, letter groups, either syllables or corresponding nonsyllable groups, were presented in nonoverlapping locations at various rates; that is, the groups were isolated in both space and time. When the groups were presented in a horizontal row (Experiment 2), the subjects could exploit syllabic structure. When the groups were presented in a column (Experiment 3), however, syllabic structure was not used, and the overall level of word identification dropped dramatically. Combining Mewhort and Beal's evidence with the present data, it is clear that a critical factor controlling use of syllabic structure is the availability of a horizontal spatial dimension.

While the availability of the spatial dimension is a necessary condition for use of syllabic structure, it is not a sufficient one. In a bar-probe task, for example, performance reflects difficulty of spatial addressing rather than the construction and integration of derived units (e.g., Mewhort \& Campbell, 1978).

While it provides a better estimate of integration speed than that in a letter overprinting task, group overprinting does not provide a precise estimate. As noted earlier, group overprinting denies the parser information normally avalable in whole-word presentations. As a result, the units cannot parallel those generated with whole-word presentation. Instead, group 
overprinting should yield units different from the corresponding whole-word case. As a result, integration speed should suffer.

Mewhort and Campbell (1978) have shown that, over and above any difficulty with character identification, masking introduces spatial uncertainty. Because the scan-parse operator requires precise spatial information, increasing spatial uncertainty by masking should alter the output of that mechanism. If we assume that masking does alter the output of the parser, the present results fit with two other, apparently contradictory reports. Mewhort and Beal (1977, Experiment 2) documented both a stronger syllable effect and much better word identification than that found in the present experiments. In a similar study, Manelis and Atkinson (1974) reported no syllable effect.

Mewhort and Beal (1977, Experiment 2) presented syllables in nonoverlapping locations on a horizontal row. When successive syllables were presented from left to right, word identification was at ceiling and was essentially independent of the rate of presentation. In the present study, a corresponding level of performance with syllabic overprinting required an intergroup interval of about $200 \mathrm{msec}$. The difference likely reflects the impact of masking, a characteristic of the overprinting paradigm, on the parser.

Manelis and Atkinson (1974) presented syllables on nonoverlapping locations. The displays were both preceded and followed by a mask, and the syllables were isolated by concurrent masking: There was no syllable effect. If masking affects parsing, however, Manelis and Atkinson's failure to show a syllable effect is hardly surprising. ${ }^{3}$ Indeed, from our perspective, the three cases fall on a continuum: Mewhort and Beal (1977) excluded masking and showed a clear syllable effect; Manelis and Atkinson used masking to define syllables and showed no syllable effect. The present experiments fall between the extremes in both respects. In light of the continuum, it is interesting to recall Travers' (1973) result: He found masking had its major impact on integration rather than on basic letter identification.

A methodological caution follows from the link between masking and parsing. A mask is often used in conjunction with forced-choice technology to limit performance levels. Indeed, it has become popular to adjust the delay of a mask to force performance to a criterion. If the suggestion concerning the effect of masking on parsing is correct, such methodology includes features quite unlike those intended.

Throughout, our interpretation has focused on an active rule-driven unit-construction device. Alternatively, one could consider a passive feature-filter device to construct supraletter units. The present data do not distinguish the two suggestions. The active mechanism is consistent with earlier order-of-report data (e.g., Mewhort, 1974; Mewhort \& Beal, 1977), and it is hard to imagine how a passive system could explain such phenomena. Further, we doubt that a passive system could incorporate the full range of rules and include an order-of-report feature without becoming an active mechanism de facto if not de jure. Nevertheless, several investigators seem to favor the feature-filter account (e.g., Purcell, Stanovich, \& Spector, 1978). In any case, the present data show that the device follows character identification but precedes entry to short-term memory.

A final point deserves mention. Taft (1979a, 1979b) has suggested a derived-unit model using a morphologically based parsing rule. Unfortunately, he has not specified the relation of the parser to letter identification and the remaining components of the system. Nevertheless, inasmuch as he contrasts the two accounts fairly directly, his model must share the basic architecture of the Smith and Spoehr (1974) account. Briefly, Taft's account is based more on semantic considerations than on the pronunciation-based rules favored by Smith and Spoehr. For example, he suggests parsing of "persuade" and "dissuade" should strip off the prefix to leave the root "suade."

We have considerable sympathy for aspects of Taft's (1979a, 1979b) position. We feel, for example, that previous derived-unit accounts have put too much stress on purely phonological factors. Nevertheless, Taft's views are problematic. While his examples illustrate a morphologically based parsing to obtain the appropriate roots, the examples are informal. ${ }^{4}$ As he has not provided a formal set of rules, the account risks circularity: Does one have to know the meaning in order to extract the root so that one can use the root to read the word? Simultaneously, however, his empirical results present interesting puzzles for purely orthographic approaches. Further, we are not satisfied that our "extensions" to Smith and Spoehr are complete. More important, while successive revisions have improved the usefulness of the grammar, our system cannot, to date, handle all cases. ${ }^{5}$ Thus, although a variety of data implicate a derived unit, the rules for the derivation remain elusive.

\section{REFERENCE NOTE}

1. Mewhort, D. J. K., \& Campbell, A. J. Parsing and the problem of "perceptual" units. Paper presented at the meeting of the Canadian Psychological Association, Vancouver, British Columbia, June 1977.

\section{REFERENCES}

Avis, W. (Ed.). Gage Canadian dictionary. Toronto: Gage, 1973. Hansen, D., \& Rogers, T. S. An exploration of psycholinguistic units in initial reading. In K. S. Goodman (Ed.), The psycholinguistic nature of the reading process. Detroit: Wayne State University Press, 1968.

Hearty, P. J., \& Mewhort, D. J. K. Spatial localization in sequential letter displays. Canadian Journal of Psychology, 1975, 29, 348-359.

HuEy, E. B. The psychology and pedagogy of reading. Cambridge, Mass: M.I.T. Press, 1968. (Originally published, 1908.)

Kolers, P. A. Experiments in reading. Scientific American, 1972, 227(1), 84-91.

Kole rs, P. A., \& Katzman, M. T. Naming sequentially presented letters and words. Language and Speech, 1966, 9, 84-95. 
Kućera, H., \& Francis, W. N. A computational analysis of present-day American English. Providence, R.I: Brown University Press, 1967.

Manelis, L., \& Atkinson, R. C. Tachistoscopic recognition of syllabicated words. Quarterly Journal of Experimental Psychology, 1974, 26, 158-166.

MEWHORT, D. J. K. Accuracy and order of report in tachistoscopic identification. Canadian Journal of Psychology, 1974, 28, 383-398.

MEwhorT, D. J. K. DIS: An n-channel tachistoscope algorithm. Behavior Research Methods \& Instrumentation, 1978, 10, 756-760.

Mewhort, D. J. K., \& Beal, A. L. Mechanisms of word identification. Journal of Experimental Psychology: Human Perception and Performance, 1977, 3, 629-640.

Mewhort, D. J. K., \& Campbell, A. J. Processing spatial information and the selective-masking effect. Perception \& Psychophysics, 1978, 24, 93-101.

Miller, G. A. The magical number seven plus or minus two: Some limits on our capacity for processing information. Psychological Review, 1956, 63, 81-97.

Purcell, D. G., Stanovich, K. E., \& Spector, A. Visual angle and the word superiority effect. Memory \& Cognition, 1978, 6, 3-8.

Smith, E. E., \& Spoenr, K. T. The perception of printed English: A theoretical perspective. In B. H. Kantowitz (Ed.), Human information processing: Tutorials in performance and cognition. Hillsdale, N.J: Erlbaum, 1974.

SPERling, G. The information available in brief visual presentations. Psychological Monographs, 1960, 11(Whole No. 498).

TAFT, M. Lexical access via an orthographic code: The basic orthographic syllabic structure (BOSS). Journal of Verbal Learning and Verbal Behavior, 1979, 18, 21-39. (a)

TAFT, M. Recognition of affixed words and the word frequency. effect. Memory \& Cognition, 1979, 7, 263-272. (b)

Travers, J. R. The effects of forced serial processing on identification of words and random letter strings. Cognitive Psychology, 1973, 5, 109-137.

VENEZKY, R. L. The structure of English orthography. The Hague: Mouton, 1970.

\section{NOTES}

1. The hit and false alarm proportions were used to obtain $\mathrm{d}^{\prime}$ values for each subject. In the fastest group, the average $\mathrm{d}^{\prime}$ was 1.42 ; it was not possible to compute a $d^{\prime}$ for each subject in the other two groups because the false alarm rate fell to zero.

2. Travers' (1973) spread conditions are similar to the conditions used by Hearty and Mewhort (1975), Mewhort (1974), and by Mewhort and Beal (1977, Experiment 1). While the latter discussed their results in terms of the availability of good spatial information and its effect on the scan-parse operator, Travers discussed his results in terms of a global distinction, namely, the issue of parallel vs. serial processing. Travers argued that his best conditions (the spread no-mask conditions) reflect parallel processing because there was no SOA effect across a $50-$ to $200-\mathrm{msec}$ range. As shown in the forementioned studies, however, a 50-msec SOA is much too long: Mewhort and Beal (1977, Experiment 1), for example, showed a dramatic drop in word identification (from $100 \%$ to $80 \%$ ) over a 5 - to $55-\mathrm{msec}$ range of SOA. As SOA was increased from $55 \mathrm{msec}$, however, performance remained relatively stable. Rather than a question of parallel vs. serial processing, we view Travers' experiments in terms of a contrast between spatial and temporal processing.

3. Manelis and Atkinson (1974) confounded the exposure time per letter with the mask time per letter. In light of the difficulty suggested for parsing, it is interesting to note that the correlation between word identification and the informationto-noise ratio (literally, the ratio of letters to mask characters) is large $[\mathrm{r}(6)=.884, \mathrm{p}<.01]$.

4. Taft's parser strips off prefixes, but it is not always clear, from orthographic rules alone, when a putative prefix is a bona fide prefix. If "un" in "unfortunate" is a prefix, is it also a prefix in "uncle" or "under?" Indeed, the word "upon" is composed of two prefixes, "up" and "on" as in "upset" and "uprise" and "onset" and "onrush," respectively. Would his parser strip away all of the word?

5. Our silente rule fails with certain words, for example, "epitome" and "Penelope." Similarly, our consonant clustering algorithm combines "th" wrongly in, for example, "sweetheart." 